\title{
INFERENCES ABOUT THE EFFERENT SYSTEM BASED ON A PERCEPTUAL ILLUSION PRODUCED BY EYE MOVEMENTS ${ }^{1}$
}

\author{
LEON FESTINGER 2 AND A. MONTAGUE EASTON \\ New School for Social Research
}

\begin{abstract}
Precise measurement of the position of the eye as it follows a moving target makes possible the exact computation of retinal information about the path of movement of that target. Comparing this retinal information with the reported visual perception of the path of movement enables inferences to be made concerning what information about eye position was used by the perceptual system. On the assumption that information available to the perceptual system about eye position comes only from monitoring efferent commands, these inferences are also about the content of those commands. Our data and analysis suggest that the efferent command for smooth pursuit eye movement, at the stage where it is monitored, contains good information about the direction of movement but only crude information about speed.
\end{abstract}

In the last decade neurophysiologists have increasingly emphasized the importance of understanding the organization and functioning of the efferent system. The general question is how, and in what form, motor commands are formulated and issued and how the nervous system controls motor activity. Some progress has been made on such questions at a neurophysiological level. For example, studies have been reported on the relationship between firing rates in motor neurons and force exerted by limb movements (Evarts, 1966, 1968, 1972); studies have identified cells in the central nervous system that regularly fire in connection with specific motor movement (Bizzi, 1968; Bizzi \& Schiller, 1970); advances have been made in the understanding of how muscle spindles function in fine regulation of movement (Granit, 1970; Matthews, 1964). It is not our intention here to review this material but only to point out the nature of some of the questions that

1 The research on which this article is based was supported by Grant MH-16327 from the National Institute of Mental Health to L. Festinger. The authors wish to acknowledge the help, during the course of experimentation and interpretation, of Saulo Sirigatti, Melvin K. Komoda, and Harold A. Sedgwick.

2 Requests for reprints should be sent to Leon Festinger, Department of Psychology, New School for Social Research, 66 West 12th Street, New York, New York 10011. have been addressed. The problems, issues, and current state of the work is well presented by Evarts, Bizzi, Burke, DeLong, and Thach (1971).

However, there has been little or no work that addresses questions about the efferent system from a psychophysical point of view. There have been studies that have shown the importance of the efferent system in enabling adaptation to distorted visual input (Festinger, Burnham, Ono, \& Bamber, 1967; Held, 1961) but this work only implicates the efferent system-it does not explicate it. We think that it is possible to make progress in understanding the nature and functioning of the efferent system through psychophysical methods. It is the purpose of this article to propose a paradigm for doing this, to present the results of a study following this paradigm, and to present the beginnings of a theoretical model, necessarily narrow in scope, that suggests itself as a result of these findings.

\section{An Approach to the Study of the EFFERENT System}

Some aspects of visual perception are particularly well suited for analyzing what happens in the efferent system. This suitability arises from the fact that the perceptual system does not have access to information about eye movements or eye position based on proprioceptive feedback from the extra- 
ocular muscles. Since there may be some who would dispute the preceding statement, it is worthwhile to briefly summarize the evidence.

The extraocular muscles in the human do contain muscle spindle receptors and tendon receptors. These are capable of transmitting information about length of the muscle or change of length (spindle receptors) and about tension or change of tension in the muscle (tendon receptors). Thus, it is possible that such information is transmitted in a way that might be used by the perceptual system. The psychophysical evidence, however, indicates that this is not the case. Brindley and Merton (1960), for example, anesthetized the surface of the eyeball and the eyelids, put an opaque cap over the cornea to eliminate retinal information, and moved the eyes of their subjects back and forth by seizing the tendon of the lateral or medial muscle with forceps. They reported that under these circumstances the subject completely lacks awareness that his eye is moving-an awareness that might be expected to exist if information from the extraocular muscles was available to the perceptual system. Brindley and Merton further reported that if the eye is held completely motionless, and the subject is told to move his eyes, the subject reports that his eye did move.

Recently Skavenski (1972) and Skavenski and Steinman (1970) have disputed this result. Among other things, they reported that if the eye is moved mechanically by large amounts $\left(6^{\circ}\right.$ to $10^{\circ}$, the subject will correctly identify the direction of the eye movement $75 \%$ to $80 \%$ of the time. One cannot be certain in their study that some clues were not available from pressure on the eyeball or on eyelids. In another study, however, Skavenski, Haddad, and Steinman (1972), using the same technique, showed conclusively that, even if any proprioceptive information from the extraocular muscles exists, it is not used by the perceptual system for egocentric localization of visual direction. They find that the only extraretinal information that is used perceptually is "outflow" information, that is, informa- tion about the efferent commands to the muscles.

We can, then, conclude that, in the absence of retinal information, knowledge about eye movements or eye position that is available to the perceptual system is based on information about what the eye was commanded to do and not on information about what the eye actually did do. At some level of transmission of an efferent command from the central nervous system, this command is monitored and the information contained in that command at that level is available to the perceptual system.

How does this help us to study the efferent system? Let us imagine a situation in which an observer watches a target moving on a completely contourless background, his eyes more or less following the movement of the target. The observer's perception of the path of movement of the target must depend on a combination of two kinds of information: information about movement of the target on the retina and information about eye movements. Only if both these sets of information were accurate, and only if they were accurately combined, would the perception of the path of movement be veridical. If the eye is stationary, of course, perception could be based only on retinal information; but with a moving eye this is not possible.

Let us tentatively assume that position and movement on the retina are accurately transmitted to the perceptual system. It seems reasonable to assume this since, if it were not so, visual perception would rarely be veridical and we know it usually is. Let us further tentatively assume that the "calculations" that combine retinal information with information about eye position are accurate. We will examine this assumption further in connection with specific data, but let us proceed now accepting the assumption.

Under this set of assumptions it is realistically possible to collect data from which one can infer the informational content of the efferent commands for eye movements as they exist at the level of the output system at which they are monitored and are available to the perceptual system. If we 
had (a) precise measurement of the perception of the path of the target moving on a contourless field, $(b)$ precise measurement of the position of the observer's eye at every moment in time while observing the target, and $(c)$ precise information of the actual position in space of the target at every moment in time, we could then perform the following computations. From the information about the actual position of the target and the actual position of the eye, we could compute the actual path traveled by the target on the retina. By comparing this retinal information with the perception of the path of movement of the target, we could infer what information the perceptual system had about the movement of the eye. This inference would, according to our reasoning, also be an inference about the informational content of the monitored efferent commands.

If the observer's perception of the path of movement were veridical, the whole endeavor would, of course, be in vain. We would simply conclude that the perceptual system had complete and accurate information about the eye movements-a conclusion that would make this line of approach not very productive. There is evidence in the literature, however, that if the eye engages in smooth pursuit movements the observer's perception is, frequently, far from veridical. Johansson (1950), for example, reported various interesting misperceptions of the paths of movement when several luminous points move in a relatively contourless field at velocities that would be expected to elicit smooth pursuit eye movements. Johansson interpreted his data differently, however, rejecting the possibility that eye movements are important in the misperceptions. As another example, Stoper (1967) reported that during smooth pursuit movements of the eye, the perception of the location of a briefly flashed spot of light is determined almost entirely by its retinal location without much "compensation" for eye movement.

It is, then, possible that the monitored efferent command for smooth pursuit eye movements does not contain complete and accurate information. The data collection procedure we outlined above might enable us to infer what information is actually avail- able. We consequently chose one such "illusion" for intensive study.

\section{The Fujii Illusion}

Fujii (1943) reported an extensive series of experiments that dealt primarily with the perception of the diameter of the circular path of a spot of light moving in an otherwise dark room. In the course of these experiments he also reported on the perceived path of motion of a spot that moved, for one cycle, in a triangular path or in a square path. In his experiments each side of the triangular path subtended a visual angle of about $17^{\circ}$ and each side of the square path subtended a visual angle of about $12.5^{\circ}$. He employed velocities of $6^{\circ}$ and $22^{\circ}$ per second. He reported a surprising difference between the actual and the perceived path of movement under these circumstances. Figure 1 (adapted from Figures 2 and 4 in Fujii, 1943) summarizes his report of these perceived paths. The solid lines indicate the physical paths, and the arrows indicate the direction of motion. The dashed lines describe the perceived paths. His report about this is very brief.

This misperception of the path of movement seemed to fit the conditions that would enable us to make inferences about the efferent output for smooth pursuit eye movements. We consequently engaged in a series of observations to see whether or not the Fujii illusion did fit the conditions and assumptions that we described above. These observations were all carried out with a spot repetitively cycling in a square path on a cathode ray tube display with a very fast phosphor so that there was no physical trace of the path of movement. A contrast screen in front of the cathode ray tube eliminated any general glow from the surface and the room was in total darkness. In short, for our observations the luminous spot moved on a contourless background.

The observations reported below are based primarily on verbal reports from many observers. All observers were asked to follow the spot with their eyes. In addition to the observations of the authors, colleagues, and graduate students in our laboratory, we also obtained reports from naive observers. 
Occasionally we encountered an observer who simply did not perceive the illusion. We did not keep a record of how many since that was not our purpose at the time. Our memory is that this amounted to 2 or 3 persons out of more than 50 observers whom we used at one time or another. The results presented below were reported by all observers who perceived the illusion. We will present these results organized around major questions.

Does the misperception of the path of movement exist under conditions in which smooth pursuit movements are likely to occur? We explored the perception of the path of movement over a wide range of sizes of the square path $\left(3^{\circ}\right.$ to $10^{\circ}$ of visual angle per side) and a wide range of frequencies ( .1 to 2.0 cycles per second) and velocities $\left(2^{\circ}\right.$ to $60^{\circ}$ per second). For these observations the spot always moved with uniform speed, turning corners instantaneously. The conclusions may be summarized as follows.

If the speed of the moving spot exceeded $40^{\circ}$ to $45^{\circ}$ per second, the misperception did not exist. Sometimes, at such high velocities the spot seemed to move erratically but the corners were perceived as right angles. At lower velocities, frequency was a critical variable. If the frequency exceeded 1.6 to 1.7 cycles per second, the illusion disappeared and the path was seen as square. Within the range of frequencies and velocities that produced the illusion, an additional observation is worth noting. At frequencies below .3 to .4 cycles per second, the perceived path of motion was usually of the form illustrated in Figure 2. At frequencies above .5 to .6 cycles per second, the perception was of the form shown in Figure 3.

In summary, we can answer the question positively. The velocities and frequencies that produce the misperception are ones that are likely to elicit some amount of smooth pursuit eye movement.

Is the retinal information accurate under these conditions? Since there are many visual illusions that do not depend on eye movements, it is important to know whether the Fujii illusion exists if the eye is stationary and all of the information about the
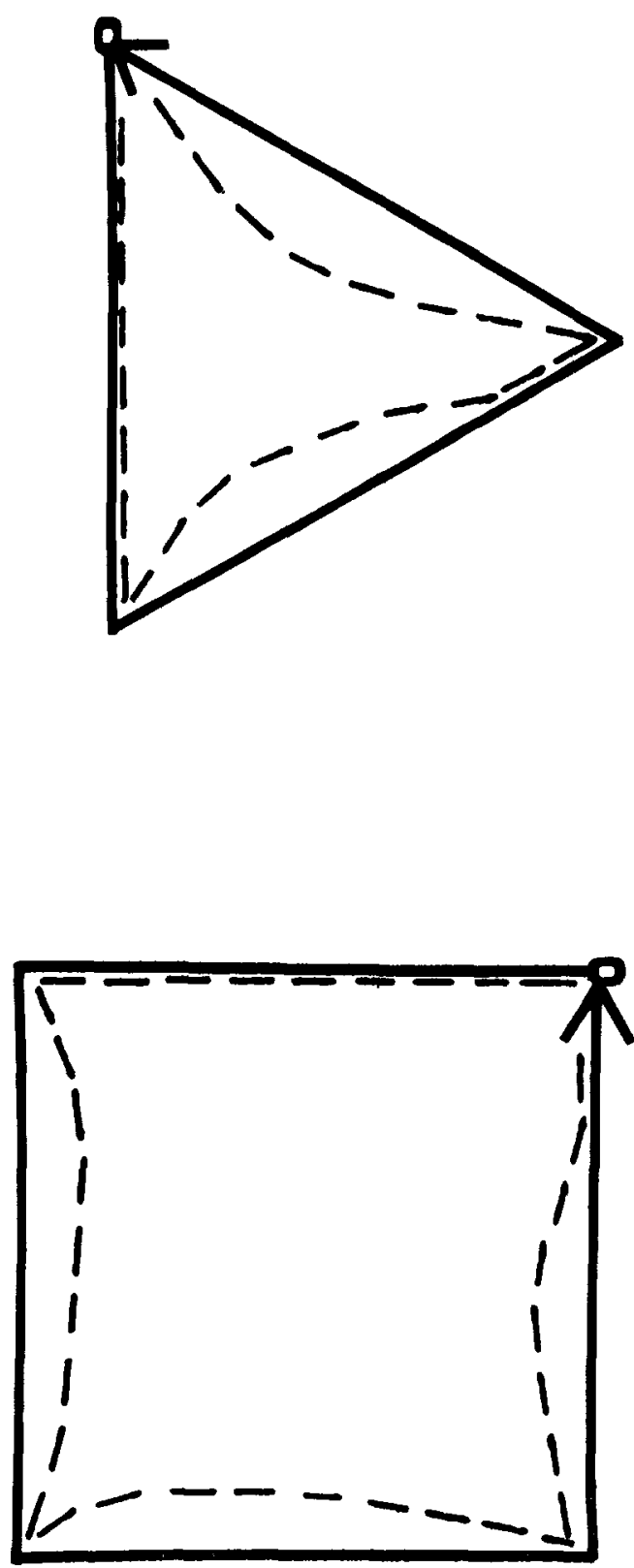

Figure 1. Perception of the path of a target moving in a square or triangular path. (Solid lines indicate the physical paths, and arrows indicate the direction of motion. Dashed lines describe the perceived paths.) (Adapted from an article by $E$. Fujii from the 1943 Japanese Journal of Psychology. Copyrighted by the Japanese Psychological Association, 1943.)

path of movement is available from the retina. The answer to this question is clear. If a fixation point is provided, and the ob- 


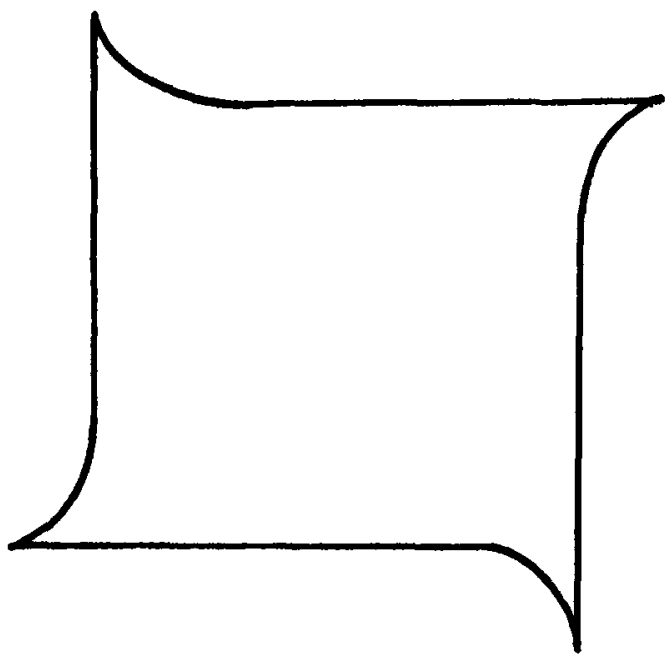

FIGURE 2. Perception of the path of a target moving in a square path at frequencies below .3 to .4 cycles per second.

server is asked to fixate that point and not follow the moving spot, the illusion disappears-the path is seen veridically as a square.

In addition, it may be pointed out that if a cathode ray tube display with a slow phosphor is used so that there is an extended physical trace of the path of movement of the spot, the path is seen as a square.

We still, however, do not have a complete answer to our question. One might suspect

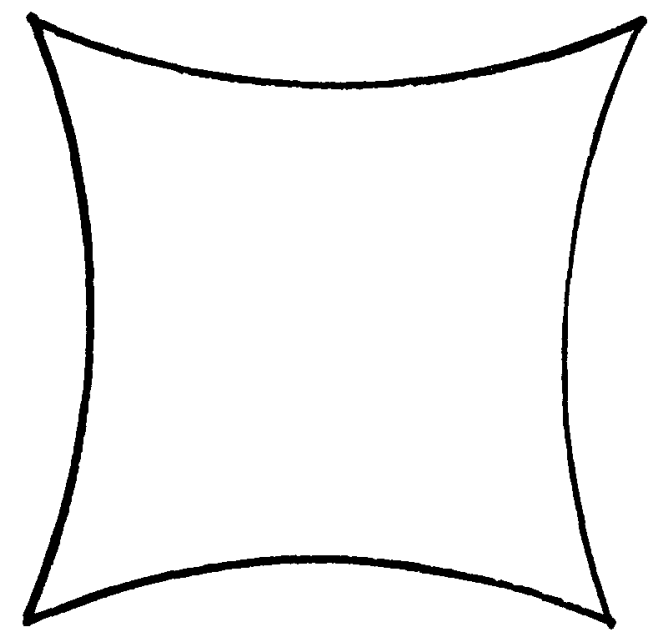

Figure 3. Perception of the path of a target moving in a square path at frequencies above .5 to .6 cycles per second. that even if the retinal information was accurate when the eye was relatively stationary, such information might be inaccurate while the eye was moving. Pursuing the hunch that the Fujii illusion might be due, at least in part, to the fact that the spot turned the corners instantaneously, a feat that the eye could not accomplish, we observed the path of movement with the eye following the spot while the spot moved with sinusoidally varying velocity along each side of the square. Under these conditions the velocity of the spot is fastest in the middle of each side, slowing up as it approaches each corner. The results are again clear. With sinusoidally varying velocity of the spot, the path of movement is seen as square. We can, then, assert that even while the eye is in motion the shape of the path of movement may be seen veridically. It may be noted that, under these conditions, the perceived square path is considerably smaller than the physical path. We will discuss this in detail later.

Is the computation that combines retinal information with information about eye position accurate? This question is, of course, least amenable to a direct answer. Since we do not know the exact information that the perceptual system has about eye position (that is what we hope to be able to infer), it is certainly difficult to show that computations involving that information are accurate. The best we can do is to show that under some circumstances, even with eye movements, perception is veridical. If the perception is veridical, it seems plausible to assume that the computation was accurate. We have already mentioned that when the spot moves with sinusoidally varying velocity, the path of movement is perceived correctly as a square. This lends weight to the assumption that the computation is accurate. Another piece of evidence can also be produced. If one provides two stationary points for fixation and asks the observer to move his eye from one to the other while the moving spot is cycling with uniform speed, one finds that again the perception is veridical. The path is seen as a square. Under these conditions, of course, the eye movements are saccades and not smooth 
pursuit movements; hence, we can at least assert that the computation for combining retinal information and information about saccadic eye movements seems to be accurate.

We may then say that, as far as we could determine, the Fujii illusion does fit the conditions that we would like to have for attempting to infer the content of the monitored efferent command for smooth pursuit eye movements. We proceeded to collect the relevant data.

\section{Measurement of Eye Position}

All observations during which eye movements were recorded were made with the observer's left eye occluded. The position of the right eye was recorded continuously, using a noncontacting eye tracker developed by Cornsweet and Crane (1972). Infrared light was projected onto the eyeball and the system measured the position of the reflection from the rear surface of the eye lens (fourth Purkinje reflection) in relation to the position of the corneal reflection (first Purkinje reflection). Two direct-current voltage outputs were obtained, one corresponding to the horizontal, the other to the vertical, component of eye position.

The output of the eye tracker has a noise level of about $2^{\prime}$ of arc and is linear (except for possible local irregularities of the observer's eye) over a greater than $12^{\circ}$ range both horizontally and vertically.

The data on eye movements were recorded in two ways. A record of the horizontal and vertical components was made, using an oscillograph, on chart paper moving at a rate of 100 millimeters per second. The two output voltages from the eye tracker were also put through two digital voltmeters used as analogue-digital converters, and the digital information was printed out once every 35 milliseconds by a high-speed printer. Event markers on both the chart paper and the digital printout indicated the time at which the moving spot in the visual display turned each corner of its square path so that the two records could be collated in time, and also, of course, to allow exact computation of the position of the eye in relation to the position of the moving target at each moment in time. The digital voltmeters used as analogue-digital converters took from 2 to 7 milliseconds from the time they received a command to sample to the time they completed the sampling: hence, there is that much uncertainty in the temporal resolution of our data. The commands to the analogue-digital converters to sample were synchronized with the movement of the spot in the visual display, so that a "sample command" always occurred at the moment the spot reached a corner of the square path.

\section{Procedure for Data Collection}

The moving spot was displayed on a HewlettPackard model 1310A display scope with a P15 phosphor. The movement of the spot was controlled by a Wavetek model 116 function generator and a Tektronix model 4701 eight-channel multiplexer. The visual display was located 100 centimeters in front of the observer's eye.

Data on eye movements while viewing the target that moved in a square path were collected from three observers. One of these (M.) was a highly experienced observer; another (T.) was inexperienced but had prior knowledge about the phenomenon; the third observer (C.) was inexperienced and totally ignorant about the phenomenon. After each observer was aligned in the apparatus, with head held steady using a biteboard, calibrations were made to determine the exact eye position measures that corresponded to positions fixated in the visual field. There is undoubtedly some error in these calibrations since the eye is not steady in fixation. We estimate this error to be no greater than $4^{\prime}$ or $5^{\prime}$ of arc.

The data were collected in total darkness. Each observer viewed four conditions of the visual display as follows: With the spot moving with uniform speed in a square path of $6^{\circ}$ per side, the length of time for the spot to traverse each side was either 350,700 , or 1,015 milliseconds. In addition each observer viewed the spot moving in a smaller square path, $3^{\circ}$ per side, the length of time to traverse each side being 350 milliseconds. The target velocities corresponding to these four conditions are, in order, approximately $18^{\circ}, 9^{\circ}, 6^{\circ}$, and $9^{\circ}$ per second. For each of these conditions, the observer fixated a stationary spot which then started to move clockwise. After eight cycles of the square the spot stopped and the observer fixated the spot again. During this entire period eye movements were recorded.

For the analysis of the data we relied primarily on the digital printout at 35-millisecond intervals. The chart paper recordings were used for two purposes: easy identification of cycles during which eyeblinks occurred, which were excluded from analysis, and easy identification of the occurrence of saccades. Sometimes the digital sampling occurred during the course of a saccade and, when this happened, we used the chart paper record in an attempt to specifiy more exactly in time the beginning and end of the saccade.

\section{Patterns of Eye Movements}

Although the plotting of the exact pattern of eye movements that occur while the observer follows the moving spot is only the first step in the calculation we wish to make, it is nevertheless important to see what they look like. We selected four examples of such eye movements to illustrate each of the 
four conditions of observation that we employed and to represent each of the three observers. These examples are shown in Figures 4, 5, 6, and 7. They are quite representative of the typical kinds of eye movements.

Each figure shows the exact position of the eye in relation to the physical location of the moving spot for one complete cycle of the square path. The small squares indicate the physical location of the corners of the square path through which the spot travels. The filled circles in each figure indicate the position of the eye at one moment in time; successive circles are separated by 35 milliseconds. The position of the eye at the moment in time that the spot instantaneously turns each corner is indicated by an open circle around that particular data point. The cycle starts at the point labeled "S" and ends

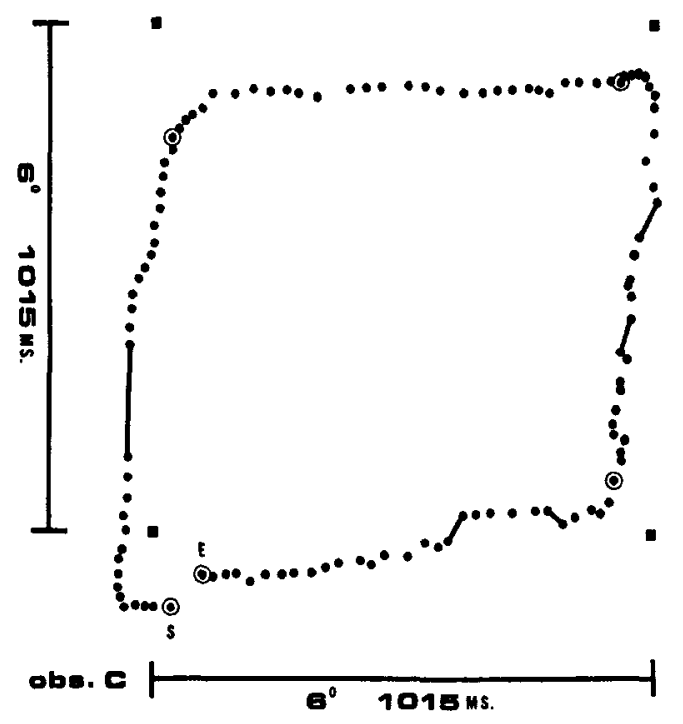

Figure 4. Successive eye positions for Observer C. for one cycle of target moving at .25 cycles per second $\left(6^{\circ}\right.$ path). (Small squares indicate the physical location of the corners of the square path. Filled circles indicate position of the eye at one moment in time; successive points are separated by 35 milliseconds. Open circles around filled circles indicate position of the eye at the moment the spot instantaneously turns the corner. The cycle starts at " $S$ " and ends at " $E$ ". Unconnected consecutive circles indicate smooth pursuit movement. Circles connected by solid lines indicate saccadic movements. Abbreviations: Obs. $=\mathrm{ob}-$ server; ms. = millsecond.)

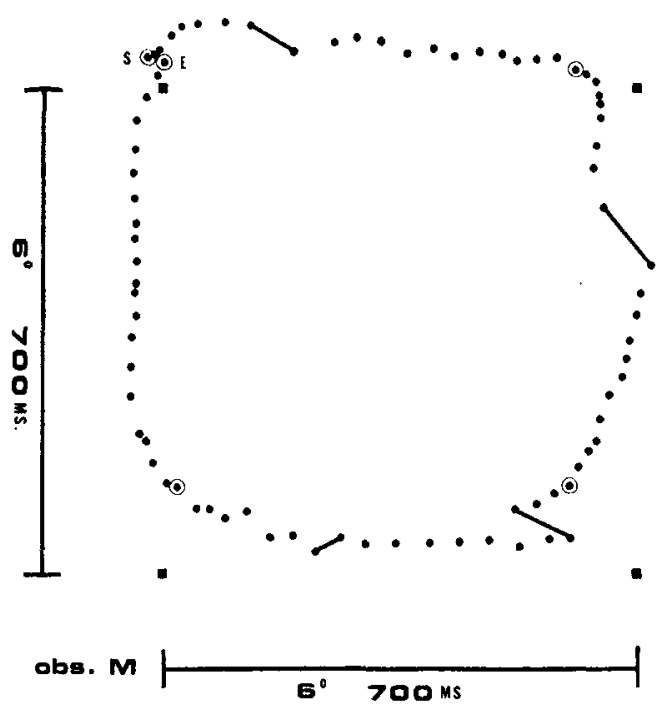

Figure 5. Successive eye positions for Observer $M$. for one cycle of target moving at .36 cycles per second $\left(6^{\circ}\right.$ path). (Small squares indicate the physical location of the corners of the square path. Filled circles indicate position of the eye at one moment in time; successive points are separated by 35 milliseconds. Open circles around filled circles indicate position of the eye at the moment the spot instantaneously turns the corner. The cycle starts as "S" and ends at " $E$." Unconnected consecutive circles indicate smooth pursuit movement. Circles connected by solid lines indicate saccadic movements. Abbreviations: Obs. $=$ observer; $\mathrm{ms}$. $=$ milliseconds.)

at the point labeled "E." If consecutive circles are not connected, that indicates that the eye was engaging in smooth pursuit movement. If the eye engaged in saccadic movements, the circles are connected by a solid line. The irregularities in the pattern of eye movements, most noticeable at the slower speed shown in Figure 4, are probably due to errors of measurement. The magnitude of these irregularities is well within the noise level and temporal resolution capability of our equipment.

It may be seen that all the eye movement patterns show a combination of smooth pursuit movements with interspersed saccades. As the velocity of the moving spot increases, the velocity of the smooth pursuit movement, of course, also increases. In addition, as the velocity increases, the interspersed saccades tend to become larger. 
There are no periods in which the eye is relatively stationary, as occurs in situations in which only saccadic eye movements are made. Here, the saccadic eye movement, when it occurs, seems to be superimposed on, and does not disturb or interrupt, the smooth pursuit movement. This is evident in all of the figures but can, perhaps, be seen most clearly in Figure 6 . In this cycle, two saccades occurred while the eye was following the spot moving upward on the left side of the square path. In each case, at the end of the saccade, the eye immediately shows smooth pursuit movement in essentially the same direction as the movement prior to the saccade. It is as if the smooth movement continued throughout with the saccade overlaid on it. It may be worth noting that Woodworth and Schlosberg (1956, p. 511) made this same point about such situations. It may also be worth

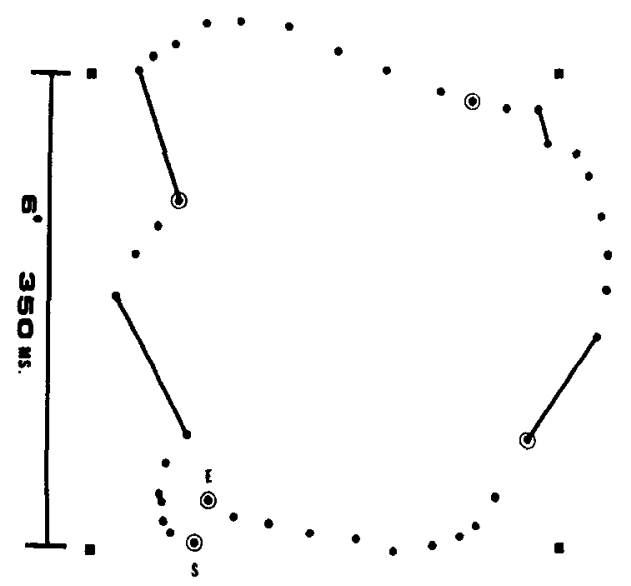

oba.c

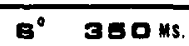

FIGURE 6. Successive eye positions for Observer $C$ for one cycle of target moving at .71 cycles per second $\left(6^{\circ}\right.$ path). (Small squares indicate the physical location of the corners of the square path. Filled circles indicate position of the eye at one moment in time; successive points are separated by 35 milliseconds. Open circles around filled circles indicate position of the eye at the moment the spot instantaneously turns the corner. The cycle starts at "S" and ends at "E." Unconnected consecutive circles indicate smooth pursuit movement. Circles connected by solid lines indicate saccadic movements. Abbreviations: Obs. $=$ observer $;$ ms. $=$ milliseconds. $)$

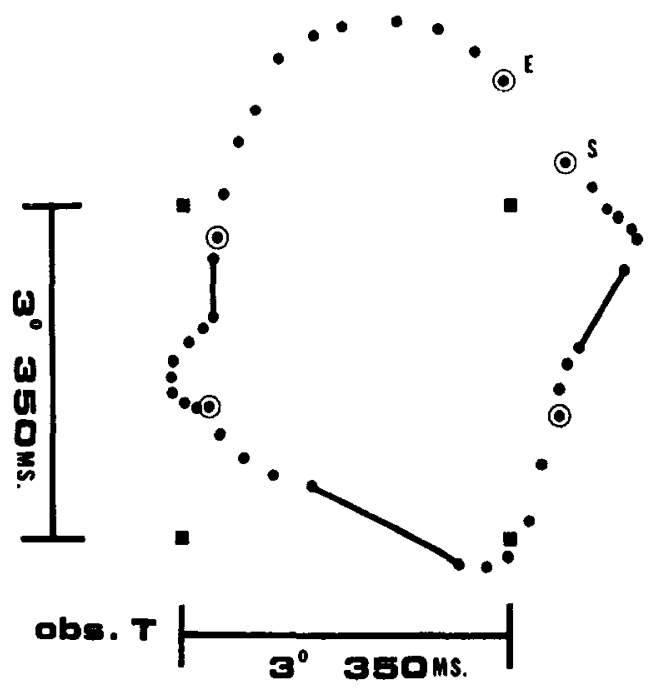

FIgURe 7. Successive eye positions for Observer $T$. for one cycle of target moving at .71 cycles per second $\left(3^{\circ}\right.$ path). (Small squares indicate the physical location of the corners of the square path. Filled circles indicate position of the eye at one moment in time; successive points are separated by 35 milliseconds. Open circles around filled circles indicate position of the eye at the moment the spot instantaneously turns the corner. The cycle starts at " $S$ " and ends at " $E$." Unconnected consecutive circles indicate smooth pursuit movement. Circles connected by solid lines indicate saccadic movements. Abbreviations: Obs. $=\mathrm{ob}-$ server; ms. $=$ milliseconds.)

noting, in passing, that there tend to be more vertically than horizontally oriented saccades. We do not find it mentioned in existing literature but, in our data, the strong suggestion exists that smooth pursuit movement in the horizontal direction is more adequate than in the vertical direction.

It is particularly relevant to our present purpose to examine, in Figures 4, 5, 6, and 7 , the pattern of eye movements around the time that the moving spot actually turns a corner of the square, that is, near the encircled data points. It is clear, in all the eye movement records, that the eye does not turn corners instantaneously as does the spot. When the spot turns a corner the eye makes a rather gradual turn with a component of motion in the previous direction persisting for some time. At higher velocities particularly, the eye sometimes, probably in anticipation, begins its gradual turn 
even before the spot has reached the corner. While the eye is making these gradual turns, of course, the movement of the spot on the retina would show an indentation from the corner similar to what is reported perceptually. This gives us our first clue as to why the misperception of the path of movement takes its particular form, namely, the indentation from the corners.

\section{Calculation of "Corrected Retinal Information"}

From the data on eye movements such as those shown in the Figures 4, 5, 6, and 7, we could, of course, compute and plot on a graph the exact movement of the spot on the retina of the observer. This would tell us what retinal information existed concerning the path of movement of the spot. This would be an adequate procedure for our purposes if the eye movement patterns consisted entirely of smooth pursuit movements. We would then be able to make inferences about what information concerning these smooth pursuit movements is available to the perceptual system.

The eye movement patterns, however, do not contain only smooth pursuit movements but also show interspersed saccades. It is necessary then, for our purposes, to make some assumptions about what information the perceptual system has about saccadic eye movements and to correct the calculation of retinal information to take this into account. In other words, we want to calculate a combination of what is known about the path of movement of the spot from retinal information and from information about saccadic eye movements. Then the only missing information would be about the smooth pursuit eye movements, and we could proceed with our inferences about that information. It is clear, of course, that the validity of these inferences will depend, in part, on the validity of our assumptions concerning information about saccades.

It seems most plausible to make the assumption that the perceptual system does have good information about the extent and direction of a saccadic eye movement. Such eye movements are very rapid and are bal- listic in nature. The efferent commands for such eye movements must, hence, be entirely preprogrammed. That is, the complete set of instructions for the movement has to be issued before the movement starts since, once the ballistic eye movement is under way, it is no longer controllable. It seems sensible to assume, then, that the monitored efferent command for such a movement contains all the information. In addition, our own informal observations indicate that if fixation points are provided, and only saccadic eye movements presumably occur, the perception is veridical. So in our calculations we will assume that, whenever a saccadic eye movement occurs, the perceptual system knows accurately and exactly the direction and magnitude of that movement.

Before we can proceed with these calculations one other decision has to be made. Do we assume that when a saccade occurs it has interrupted and replaced the smooth pursuit movement, or do we assume that the saccade is overlaid on, or added to, the smooth pursuit movement which persists? As mentioned above, particularly in reference to Figure 6 when we were discussing the patterns of eye movements, the records look as though the most plausible assumption is the latter-that the smooth purstit component of the motion of the eye persists and the saccadic movement is added on to that. In our calculations we have attributed a speed and direction of smooth pursuit movement during the interval occupied by a saccade by interpolation from the smooth pursuit movements immediately preceding and immediately following the saccade.

\section{"Corrected Retinal Paths" of the Moving Spot}

Given these assumptions we can calculate what the perception of the path of motion of the spot would be if it were based solely on information about movement on the retina and correct information about saccades that were superimposed over an ongoing smooth pursuit motion. Representative examples of the results of these calculations are presented in Figures 8, 9, 10, and 11. They are chosen, again, to show one example from 

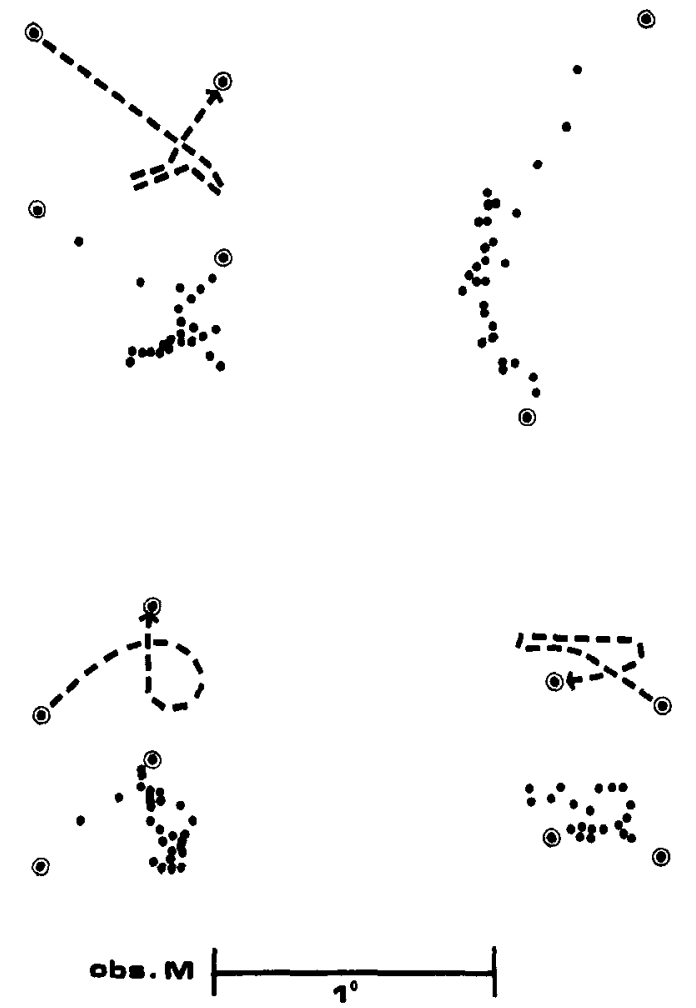

Figure 8. Corrected retinal path for Observer $\mathrm{M}$. for one cycle of target moving at .25 cycles per second $\left(6^{\circ}\right.$ path). (Each filled circle represents successive relative positions at 35 -millisecond intervals of the moving spot on the retina, plotted in terms of visual field rather than the reversed retinal field, corrected for saccadic eye movements. Encircled circles indicate retinal position of the spot at the moment it turns a corner. For visual clarity, the retinal path for each side of the square is separated from the others. Dashed line indicates general path on retina where data points were clustered very closely. Abbreviation: Obs. = observer.)

each of our four observation conditions and at least one example from each of our three observers. These figures are quite typical of all of the data.

Each of the figures shows the "corrected retinal path" for a single cycle of the eye following the spot. Each filled circle again represents successive relative positions (35millisecond intervals) of the moving spot on the retina (plotted in terms of visual field rather than the reversed retinal field) corrected for saccadic eye movements. The encircled data points again indicate retinal position of the spot at the moment that it turns a corner. For the sake of visual clarity, the retinal path for each side of the square is separated from the others. It may be remembered that the direction of target movement is always clockwise. On each figure is indicated the scale equivalent of $1^{\circ}$ of visual angle. At the lowest frequency, illustrated in Figure 8, many of the data points cluster so closely that the path on the retina is not readily apparent. Where this is the case we have indicated, with a dashed line, the general path on the retina.

Let us first point to the most salient features of Figures 8, 9, 10, and 11. It is clear that in many instances they conform to the general features of the reported perceptions of the path of movement of the spot. At the lower frequencies, illustrated in Figures 8 and 9, the typical pattern along one side of the path is a rapid inward inden-

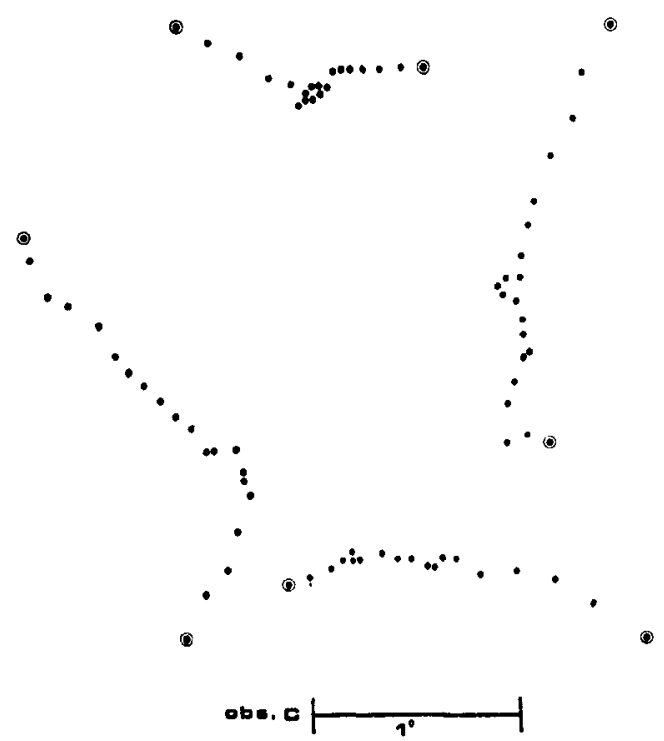

Figure 9. Corrected retinal path for Observer $C$. for one cycle of target moving at .36 cycles per second $\left(6^{\circ}\right.$ path). (Each filled circle represents successive relative positions at 35 -millisecond intervals of the moving spot on the retina, plotted in terms of visual field rather than the reversed retinal field, corrected for saccadic eye movements. Encircled circles indicate retinal position of the spot at the moment it turns a corner. For visual clarity, the retinal path for each side of the square is separated from the others. Abbreviation: Obs. $=$ observer.) 

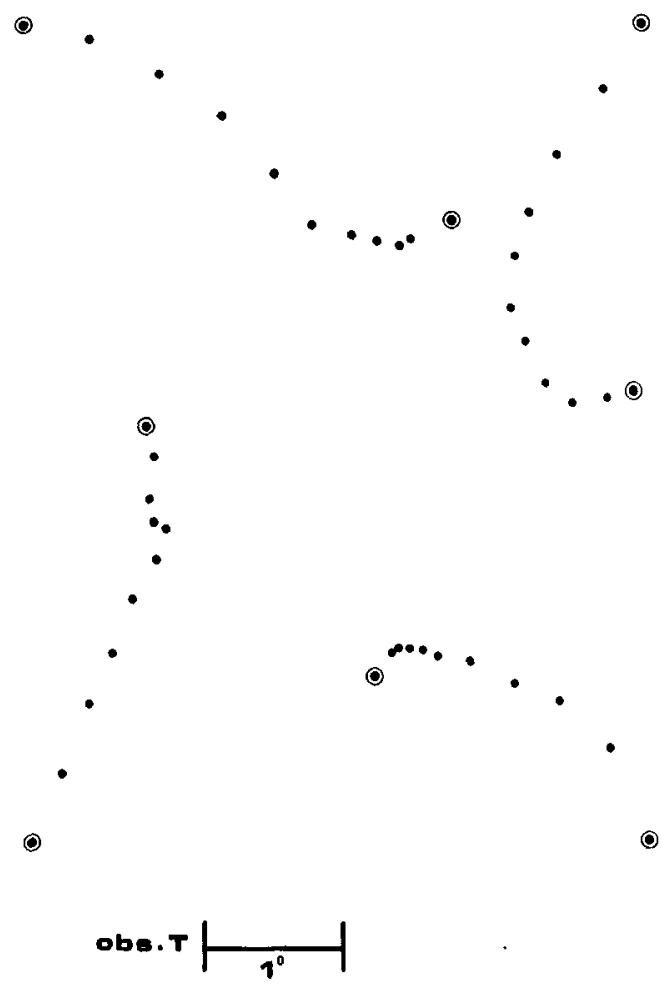

Figure 10. Corrected retinal path for Observer $\mathrm{T}$. for one cycle of target moving at .71 cycles per second $\left(6^{\circ}\right.$ path). (Each filled circle represents successive relative positions at 35 -millisecond intervals of the moving spot on the retina, plotted in terms of visual field rather than the reversed retinal field, corrected for saccadic eye movements. Encircled circles indicate retinal position of the spot at the moment it turns a corner. For visual clarity, the retinal path for each side of the square is separated from the others. Abbreviation: Obs. = observer.)

tation from the corner point, followed either by a slow stretching out of the path until the next corner, as in Figure 9, or by a close clustering of points, as in Figure 8 . The best illustrations of this are, perhaps, the path on the left-hand side of Figure 8 and on the lower side of Figure 9.

At the higher frequencies, illustrated in Figures 10 and 11, the corrected retinal paths look much more symmetrically indented, conforming to the general form of the perception shown in Figure 3. The best examples of this are the right-hand side of Figure 10 and several sides in Figure 11. It may be of interest to note that Observer
T., whose data are shown in Figure 10, often reported the perception of asymmetrical sides even at higher frequencies.

It is clear from an examination of Figures $8,9,10$, and 11 that the information contained in these corrected retinal paths provides a major basis for the Fujii illusion. One can readily come to the conclusion that the information available to the perceptual system about the position of the eye during smooth pursuit movement is not very great. Our purpose, however, is to try to infer as much as we can about exactly what information is available about smooth pursuit movements; hence, our next step should be to examine the major ways in which these patterns of corrected retinal path differ from the reported perceptions. There are two

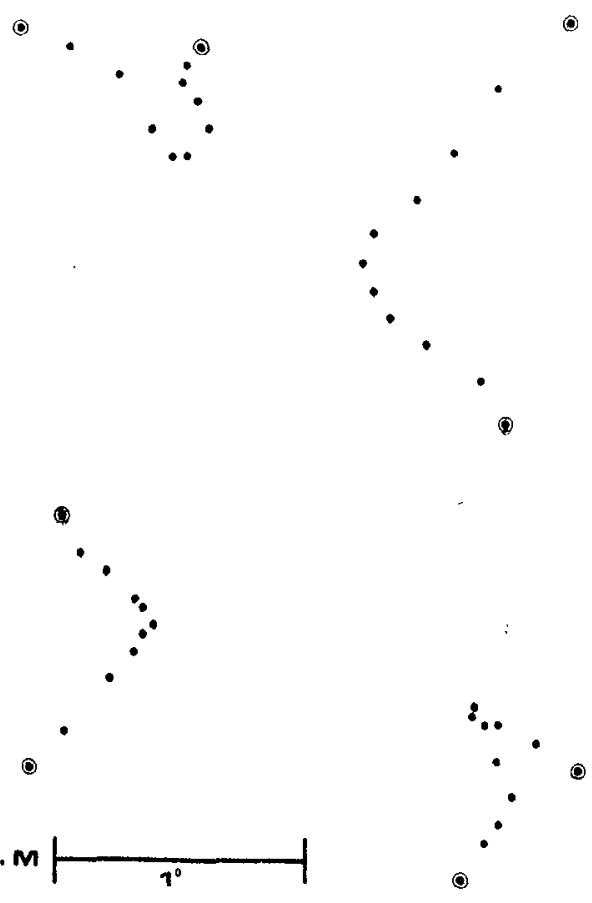

Figure 11. Corrected retinal path for Observer M. for one cycle of target moving at .71 cycles per second $\left(3^{\circ}\right.$ path). (Each filled circle represents successive relative positions at 35 -millisecond intervals of the moving spot on the retina, plotted in terms of visual field rather than the reversed retinal field, corrected for saccadic eye movements. Encircled circles indicate retinal position of the spot at the moment it turns a corner. For visual clarity, the retinal path for each sicle of the square is separated from the others. Abbrevation: Obs. = observer.) 
major differences that are important to point out.

Let us consider the corrected retinal paths shown in Figure 8. It is clear that they all differ considerably from the perception of the path of movement of the spot. Never did this observer, or any other observer, report seeing the moving spot circle back or reverse direction along any one side. These data points must, obviously, be stretched out in space in a temporal sequence if they are to conform more closely to the actual perception. In other words, the perceptual system does add something about velocity of smooth pursuit eye movement into the perception.

The same point can be made again, on a slightly different basis, considering any of the figures showing the corrected retinal paths. (Figures 8, 9, 10, and 11). For example, these paths in Figure 9 would indicate that the perceived path would have an apparent extent of about $2^{\circ}$ of visual angle per side on the average. This, again, does not correspond to the perception. Although we tried, we were not successful in obtaining precise estimates of the perceived extent from our observers. It was a very difficult thing for them to report on.

The most successful procedure was to have the observer follow the moving spot for a number of cycles and then to suddenly paint the entire square on the cathode ray tube display. One could then obtain gross comparisons of the perceived size and the actual size. What can be said from such reports is that at lower frequencies the extent of the perceived path usually looked smaller than at higher frequencies; that the perceived extent of motion was always less than the actual physical extent; and that rarely did the perceived extent seem less than about half of the physical extent. The corrected retinal paths shown in Figures 8, 9, 10 , and 11 are almost all too short. Again we must come to the conclusion that the perception has had something added in about the position of the eye during smooth pursuit movements.

The second major difference between the corrected retinal paths and the perceptions of the observers concerns the directional orientation of the paths. This can be illustrated by examining the paths in Figure 11. For this observer, as well as for all others, the perceptions are oriented correctly as they are shown in Figures 2 and 3 . But the corrected retinal paths are frequently tilted. This orientation difference does not always appear, but as can be seen in Figures 8, 9, 10 , and 11 , it frequently does exist. We are consequently led to the conclusion that the perceptual system knows something about the direction in which the eye was commanded to move during smooth pursuit.

\section{Content of the Efferent Command for Smooth Pursuit Eye Movement}

We previously stated, with support from the research literature, that whatever information the perceptual system has about the smooth pursuit movement of the eye must represent the information contained in the efferent commands for those movements at the level at which those commands are monitored. Our task now is to infer, as precisely as we can, what this exact information is. We have already said that the information cannot be very exact and specific-the corrected retinal paths are too similar to the perceptions for this to be the case. We have also said that some information about movement of the eye must be present-the corrected retinal paths need to be stretched out in temporal order; and that information about the commanded direction of the eye movement must be present-the perceived orientation of the paths was not tilted as are many of the corrected retinal paths.

If we had been successful in obtaining very precise measurement of the perception of the path of the moving spot, we would have an easier task at this point. However, we were not successful at this. We were not able to get adequate measures of the magnitude of perceived indentation from the corners, or of the perceived extent of movement of the spot. On the basis of the general reports of perception that we obtained it seems reasonable, however, to make the following inferences:

1. The efferent command for a smooth pursuit eye movement contains specification 
of the direction for the eye to move. Any instance in which the corrected retinal path does not correspond to the physical path, but the perception is veridical, must indicate that the efferent command contained correct information about the relevant aspect of the eye movement. The correctly perceived orientation of the path indicates that there must be some information about direction in which the eye is commanded to move. This does not, of course, mean that the perceptual system knows the direction in which the eye is actually moving. It only knows the direction in which the eye was commanded to move. In the particular instance of the Fujii illusion, since the eye cannot turn corners suddenly, the actual directions of eye movements probably differ from what the eye was commanded to do in the neighborhood of the corners of the path. This accounts for the perceived indentations from the corners that are reflected in the corrected retinal paths. The perceptual system only knows the direction contained in the efferent command.

2. The efferent command for a smooth pursuit eye movement contains very inadequate information about the speed of the eye movement. Since the extent of the perceived path was always considerably less than the physical extent of movement, we know that the efferent command does not contain accurate information about speed of eye movement. On the other hand, as we discussed in the previous section, some information about this is present. It must be that the actual speed of the eye movement is controlled by some more peripheral loop in the efferent output system so that the speed of the eye can be approximately matched to the speed of the target even though the command, at the monitored level, does not contain this information.

We cannot say more about this with great confidence at this point but, in speculation, we would propose the following. The efferent command for smooth pursuit movement, in addition to specifying the direction for that movement, contains only the command to move. Hence, the perceptual system knows that the eye is moving and will attribute at least some minimal velocity to that movement. When the target changes direction suddenly a new command will be issued which, in addition to ordering a direction change, may contain instructions to move faster or slower. Thus, the perceptual system could know that, at different parts of the path, the speed of the eye differed as instructed. This is our guess. Further explorations are needed in situations in which the perception can be measured more exactly.

\section{Possibility of an Alternative Interpretation}

The validity of our conclusions about the efferent output for smooth pursuit eye movements rests, obviously, on a chain of reasoning that includes a number of assumptions. It is important, consequently, to consider other possible interpretations of the Fujii illusion and the data we have collected concerning it. A successful alternative interpretation would cast serious doubt on our analysis.

There is a major possible alternative that has been suggested to us many times. This alternative would hold that the perceptual system does have accurate information about eye position during smooth pursuit movements, but that there is some time mismatch between retinal information and information about eye position. Perhaps the retinal information arrives at some central processing stage sometime later (or earlier) than the eye position information. Then the retinal information might be matched to the later (or earlier) eye position, and this temporal mismatch might produce the misperception of path of movement rather than inadequate information about eye position, as we have argued.

We can, of course, take our records of eye movements, from which we know the exact position of the eye and the position of the target on the retina at each point in time, and compute what the predicted perception would be for various assumed differences of arrival times of the two pieces of information. We have done this and in no instance does the perception predicted in this manner at all resemble the Fujii illusion. Figure 12 shows examples of the results of these computations to illustrate the out- 
come. In this figure the upper set of circles, labeled A, shows the result of our computation of corrected retinal path for Observer C. along the upper side of the $6^{\circ}$ square path, the spot taking 350 milliseconds to traverse each side. The next two sets of circles, $\mathrm{B}$ and $\mathrm{C}$, show the results of calculations that assume eye position is accurately known, and assume that the retinal information arrives 70 milliseconds earlier (B) and 70 milliseconds later $(\mathrm{C})$ than the information about eye position. The fourth set of circles (D) represents the eye and target positions from which the above calculations were made. These examples are quite typical of these computations. They do not resemble the perceived Fujii illusion. This particular alternative interpretation does not seem viable.

\section{SUMMARY}

When an observer's eyes follow a target moving on a contourless background, his perception of the path through which the target moved has to be based on a combination of retinal information and information about eye position. Precise measurement of the eye position as it follows the target can provide exact computation of what information was available on the retina. A comparison of this retinal information with the reported visual perception of the path of movement enables inferences to be made concerning what information about eye position was used by the perceptual system.

Such observations and computations were made using a situation in which the path of the moving target is known to be misperceived. On the assumption that the perceptual system does have accurate information about saccadic eye movements, some conclusions could be reached about what the perceptual system knows about smooth pursuit eye movements.

Since existing data point to the fact that the perceptual system does not have information about eye position based on feedback from the extraocular muscles, it seems that whatever information about eye position is known is based on monitoring the efferent commands issued to those muscles. Thus,
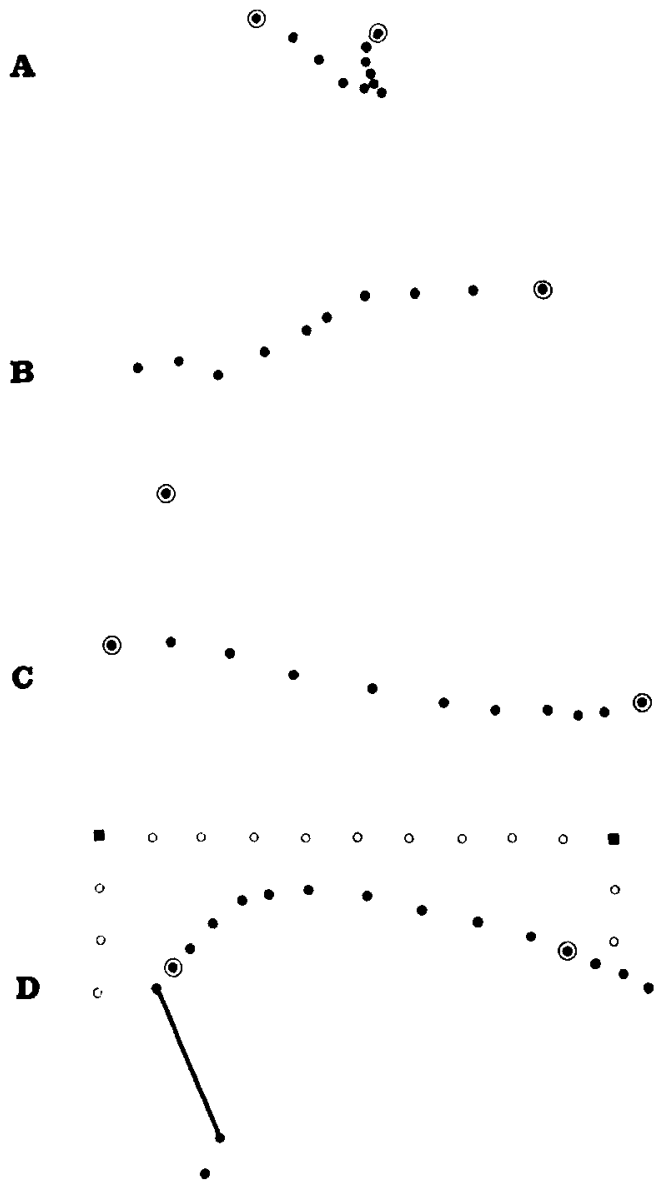

FIgURE 12. Comparison with computations based on differential latencies of retinal and eye-position information. (Section A shows corrected retinal path; Section B, eye-position information delayed 70 milliseconds; Section $C$, retinal information delayed 70 milliseconds; Section $D$, eye and target positions used in computations. Small squares indicate physical location of the corners of the path. Open circles indicate target positions, filled circles eye positions when the spot turns the corner. Solid line indicates saccadic eye movements.)

inferences and conclusions about what the perceptual system knows about smooth pursuit eye movements may also be regarded as inferences about the content of the efferent commands for such movements at that point in the efferent system at which those commands are monitored.

Our data and analysis suggest that the efferent command for a smooth pursuit eye movement, where it is monitored, contains 
information about the direction of movement but does not contain precise information about the velocity of the movement.

\section{REFERENCES}

Brzzi, E. Discharge of frontal eye field neurons during saccadic and following eye movements in unanesthetized monkeys. Experimental Brain Research, 1968, 6, 69-80.

Brzzi, E., \& Schiller, P. H. Single unit activity in the frontal eye fields of unanesthetized monkeys during eye and head movement. Experimental Brain Research, 1970, 10, 151-158.

Brindley, G. S., \& Merton, P. A. The absence of position sense in the human eye. Journal of Physiology, 1960, 153, 127-130.

Cornsweet, T. N., \& Crane, H. D. An accurate treo dimensional eye tracker using first and fourth Purkinje images. Menlo Park, Calif.: Stanford Research Institute, 1972.

Evarts, E. V. Pyramidal tract activity associated with a conditioned hand movement in the monkey. Journal of Neurophysiology, 1966, 29, 1011-1027.

EvarTs, E. V. Relation of pyramidal tract activity to force exerted during voluntary movement. Journal of Neurophysiology, 1968, 31, 14-27.

EvarTs, E. V. Activity of motor cortex neurons in association with learned movement. International Journal of Neuroscience, 1972, 3, 113-124.

Evarts, E. V., Brzzi, E., Burke, R. E., DeLong, M., \& ThACH, W. T., JR. Central control of movement. Neurosciences Research Program Bulletin, 1971, No. 9.
Festinger, L., Burnham, C. A., Ono, H., \& BAmber, D. Efference and the conscious experience of perception. Journal of Experimental Psychology Monograph, 1967, 74(4, Pt. 2).

FuJIr, E. Forming a figure by movement of a luminous point. Japanese Journal of Psychology, 1943, 18, 196-232.

Granit, R. The basis of motor control. New York: Academic Press, 1970.

Held, R. Exposure-history as a factor in maintaining stability of perception and coordination. Journal of Nervous and Mental Disease, 1961, 132, 26-32.

Jorinsson, G. Configurations in event perception. Uppsala, Sweden: Almquist \& Wicksell, 1950.

Mattiews, P. B. C. Muscle spindles and their motor control. Physiological Reviczes, 1964, 44, 219-288.

SkAvenski, A. A. Inflow as a source of extraretinal eye position information. Vision $R e$ search, 1972, 12, 221-229.

Skavensi, A. A., Haddad, G., \& Steinman, R. $M$. The extraretinal signal for the visual perception of direction. Perception \& Psychophysics, $1972,11,287-290$.

Skavensmi, A. A., \& Steinman, R. M. Control of eye position in the dark. Vision Research, 1970, 10, 193-203.

STOPER, A. E. Vision during pursuit movement: The role of oculomotor information. Unpublished doctoral dissertation, Brandeis University, 1967.

Woodwortit, R. S., \& Schlosberg, H. Experimental psychology. New York: Holt, 1956.

(Received May 29, 1973) 